\title{
Steric blockage of lysenin toxin by crowding
}

\author{
Ignacio L.B. Munguira and Alfonso Barbas
}

1 U1006 INSERM, Université Aix-Marseille, Parc Scientifique et Technologique de Luminy, 163 avenue de Luminy, 13009 Marseille, France 2 Instituto de Fusión Nuclear, José Gutiérrez Abascal 2, 28006 Madrid, Spain

bioRxiv has withdrawn this manuscript in response to an institutional investigation by INSERM. 